\title{
Forced Expression of Hnflb/Foxa3 Promotes Hepatic Fate of Embryonic Stem Cells
}

Neda Yahoo ${ }^{1}$, Behshad Pournasr ${ }^{2}$, Jalal Rostamzadeh ${ }^{3}$, Mohammad Saeed Hakhamaneshi $^{1}$, Asghar Ebadifar ${ }^{4}$, Fardin Fathi ${ }^{*}$, Hossein Baharvand ${ }^{2,5^{*}}$

1. Cellular and Molecular Research Center, Kurdistan University of Medical Sciences, Sanandaj, Iran.

2. Department of Stem Cells and Developmental Biology, Cell Science Research Center, Royan Institute for Stem Cell Biology and Technology, ACECR, Tehran, Iran.

3. Department of Biological Sciences and Biotechnology, School of Science, University of Kurdistan, Sanandaj, Iran.

4. Dentofacial Deformities Research Center, Shahid Beheshti University of Medical Sciences, Tehran, Iran.

5. Department of Developmental Biology, University of Science and Culture, ACECR, Tehran, Iran.

\section{*Correspondence:}

Baharvand@RoyanInstitute.org (H.B.) or farfath@gmail.com (F.F.)

Running title: Hnflb/Foxa3 induce hepatic fate in ES cells

Competing financial interests: The authors declare they have no competing financial interests. 


\begin{abstract}
Embryonic stem (ES) cell-derived hepatocytes have the potential to be used for basic research, regenerative medicine, and drug discovery. Recent reports demonstrated that in addition to conventional differentiation inducers such as chemical compounds and cytokines, overexpression of lineage-specific transcription factors could induce ES cells to differentiate to a hepatic fate. Here, we hypothesized that lentivirus-mediated inducible expression of hepatic lineage transcription factors could enhance mouse ES cells to hepatocyte-like cells. We screened the effects of candidate transcription factors Hnflb, Hnfla, Hnf4a, Foxal, Foxa3 and Hex, and determined that the combination of Hnflb/Foxa3 promoted expression of several hepatic lineage-specific markers and proteins, in addition to glycogen storage, ICG uptake, and secretion of albumin and urea. The differentiated cells were engraftable and expressed albumin when transplanted into a carbon tetrachloride-injured mouse model. These results demonstrated the crucial role of Hnflb and Foxa3 in hepatogenesis in vitro and provided a valuable tool for the efficient differentiation of HLCs from ES cells.
\end{abstract}

Keywords: Embryonic stem cells; Forced expression; Hepatic-lineage induction; Hepatic transcription factors. 


\section{Introduction}

Embryonic stem (ES) cells, due to their unlimited self-renewal capacities and diverse differentiation abilities [1], are expected to provide an unlimited source of cells for various applications. ES cell-derived hepatocyte-like cells (HLCs) can be used for basic research, regenerative medicine, and drug discovery [2,3]. Numerous studies have substantially contributed to the differentiation of ES cells into hepatocytes by reporting continual improvement of growth factors, cell-matrix reconstruction, cell-cell interactions, and genetic or epigenetic modifications (for review see [4]). Another approach for hepatocyte generation in vitro is the direct conversion or trans-differentiation of somatic cells to induced hepatocyte-like cells (HLCs) with combinations of different transcription factors [5-10] under hepatic conditions. Most of these differentiation protocols attempt to recapitulate liver development within a few days of the in vitro culture.

Over the last few years a number of transcription factors have been identified that have pivotal roles in liver development $[11,12]$. Therefore, it can be speculated that certain specific transcription factors or a combination of them have the capability to facilitate differentiation or direct reprogramming of ES cells into HLCs. Recently, it has been demonstrated that transcription factors or micRNAs which constitutively or transiently express in ES cells could induce hepatic differentiation in addition to conventional differentiation methods [13-19].

We hypothesized that the transient expression of hepatic lineage transcription factors could positively influence and expedite directed differentiation of ES cells into HLCs. In order to prove our hypotheses, we first constructed mouse ES cells that inducibly expressed hepatocyte nuclear factors (Hnflb, Hnfla, Hnf4a), forkhead box (Foxal, Foxa3) and Hex via lentivirus expression vectors. Next, we screened for effective transcription factors and determined that the forced expression of combined Hnflb/Foxa3 led to hepatocyte 
progenitors (HP) in hepatic medium. The results indicated that this forced expression of Hnflb/Foxa3 enhanced hepatic marker expressions, improved functionality of HLCs, and engraftment after transplantation of HPs into mice with carbon tetrachloride-induced acute liver damage.

\section{Materials and methods}

\subsection{Mouse embryonic stem cell culture, differentiation and viral transduction}

We cultured mouse embryonic stem (ES) cells (RB20) [20] under standard conditions in 2i [21]. Differentiation was induced by exogenous gene expression in presence of $5 \mu \mathrm{g} / \mathrm{ml}$ doxycycline (Dox) to the basal medium for 7 days. Later, the basal medium was supplemented with $40 \mathrm{ng} / \mathrm{ml}$ fibroblast growth factor 4 (FGF4) and $40 \mathrm{ng} / \mathrm{ml}$ bone morphogenetic protein 4 (BMP4) for 3 days, followed by $25 \mathrm{ng} / \mathrm{ml}$ hepatocyte growth factor (HGF), $10^{-7} \mathrm{M}$ dexamethasone (Dex), $1 \%$ insulin-transferrin-selenium (ITS), and $5 \mathrm{mM}$ nicotinamide for an additional 3 days. Finally, the resultant cells were replated on Matrigelcoated dishes in hepatocyte culture medium (HCM) supplemented with $25 \mathrm{ng} / \mathrm{ml} \mathrm{HGF,} 10^{-7}$ M Dex, and $50 \mathrm{ng} / \mathrm{ml}$ oncostatin M (OSM) for 10 days.

LR reactions between the entry clones of the genes of interest and the pLIX403 destination vector were performed in order to obtain the lentiviral expression vectors. The resultant vectors were transfected to $293 \mathrm{~T}$ cells in conjunction with packaging plasmid psPAX2 and envelop plasmid pMD2.G to produce lentiviruses. Viral supernatant was collected at 24 and $48 \mathrm{~h}$ after transfection. For transduction we transfected the ES cells in ES medium with lentiviruses. At $48 \mathrm{~h}$ post-transfection, the cells were subjected to puromycin $(0.2 \mu \mathrm{g} / \mathrm{ml})$ to select the transduced cells. 
All the procedures including cell cultures, viral transduction, immunostaining, quantitative RT-PCR (qRT-PCR), and functional analyses have been described in details in supplementary Materials and methods.

\section{Results}

\subsection{Screening for hepatic lineage-inducing factors in ES cells led to finding $\mathrm{Hnflb}$ and}

\section{Foxa3}

In order to transfer transcription factor genes to mouse ES cells, we used a tetracycline (tet) inducible-lentiviral system. Initially, we performed the LR recombination reaction by Gateway to generate an expression plasmid that contained the gene of interest for each factor. Using this system, we have constructed pTRE-GOI-PGK-PuroR-2A-rtTA, in which the PGK promoter constitutively drives reverse tetracycline-controlled transactivator (rtTA) expression. In the Tet-On system, expression of gene of interest (GOI) is correlated with a Tet analog, Dox. In the presence of Dox, rtTA binds to the tetracycline response element (TRE) promoter and induces expression of GOI. In the absence of Dox, rtTA is unable to activate the system (Fig. 1A). Lentivirus particles were produced by co-transfection of 293T cells with the expression construct and the packaging plasmid mix pMD2.G that consisted of $V S V-G$ and psPAX2 which contained gag/pol. We transduced ES cells with lentiviruses to generate cells that expressed individual transcription factors under Dox culture conditions. Six transcription factors (Hnflb, Hnfla, Hnf4a, Foxal, Foxa3 and Hex) were transduced into mouse ES cells. The genes were selected based on their critical role for embryonic liver development and function - Foxal and Foxa3 as pioneer factors [22]; Hex and Hnflb as hepatic specification factors [23, 24]; and Hnfla and Hnf4a as mature hepatic regulators [25, 26]. After $48 \mathrm{~h}$, we applied puromycin to the ES cell medium in order to select successfully transduced cells. Puromycin selected colonies were expanded by passaging. The 
immunofluorescence assay for Oct4 confirmed that transduced ES cells maintained their pluripotency state after transduction (Supplementary Fig. 1A). Then RT-PCR analysis was performed to confirm the exogenous gene expressions in transduced cells which cultured in Dox-containing medium for 7 days (Supplementary Fig. 1B).

To examine the effect of ectopic expression of transcription factor on hepatic gene activation, the transcription factors were overexpressed for 7 days in basal medium in the presence of Dox in an adherent culture model which was feeder free and contained low serum, followed by 6 days by withdrawing Dox in the same medium. RT-PCR analysis at day 7 indicated that forced expression of Hnflb induced expression of Foxa2, a potent early hepatic regulator (Fig. 1B). Expression analysis at day 13 also showed a 5-20 fold upregulation of $A l b$ with Foxa3 cells which has demonstrated a higher effect (Fig. 1C). Foxa3 is the most expressed Foxa family member in the adult liver [27].

We analyzed the effects of inducible expression of the factors on hepatic differentiation of ES cells in hepatic/inductive medium by culturing the transduced cells for 7 days in basal medium in the presence of Dox. Then, the enforced cells were treated with BMP4 and FGF4 for three days, followed by HGF, ITS, Dex, and nicotinamide for another three days to induce hepatic specification (Fig. 1D). Hnfla and Hnflb overexpressing cells showed a significant higher $A l b$ expression in hepatic/inductive medium (Fig. 1E). Under these induction conditions, the morphology of mouse ES cells gradually changed in the two types of Hnfla or $H n f 1 b$ cells into an extension pattern of short-sided polygons with prominent nuclei (Fig. 1F). Interestingly, a portion of these cells in the Hnfla or Hnflb cells were Alb- and cytokeratin 19 (Ck19)-positive (Fig. 1G).

In order to examine the cooperative effect of Hnflb, Hnfla, and Foxa3 on the expression of hepatic markers, we constructed double lines in which two transcription factors (Hnflb/Hnfla, Hnflb/Foxa3, or Hnfla/Foxa3) overexpressed for one week in basal medium 
in the presence of Dox. At day 13, the expressions of Alb, as well as endogenous Hnfla and Hnf4a were evaluated. RT-PCR analysis indicated that Hnflb/Foxa3 had the most upregulation of the liver specific transcription factors (Fig. 1H). Therefore, the combination of enforced Hnflb/Foxa3 induced hepatic lineage specification from ES cells even in basal medium.

\subsection{Overexpression of Hnflb/Foxa3 in ES cells under hepatic medium generated a hepatic fate}

In the next step, we evaluated the effect of forced expression of Hnflb/Foxa3 on hepatic differentiation in the presence of hepatic medium. Hnflb/Foxa3 ES cells were induced to forced expression by Dox treatment for one week and culture in hepatic medium for 6 days as previously described (Fig. 1D). At day 13, the cells exhibited morphologic changes to form colonies that contained epithelial like cells with an extension pattern of short-sided polygons and prominent nuclei (Fig. 2A). Gene expression analysis showed that HP markers Alb, alpha-phetoprotein (Afp), transthyretin (Ttr), Hnf4a, and delta-like 1 homolog (Dlk1) significantly upregulated, but not in the Dox negative control cells (Fig. 2B). We observed co-expression of Alb/Ck19 and Afp/Hnf4a at the protein level using immunofluorescence staining (Fig. 2C). Flow cytometry analysis demonstrated that $35.5 \pm 6.0 \%$ of the overexpressed cells expressed Alb whereas the control group only expressed $12.5 \pm 2.0 \%$ (Fig. 2D, $P<0.01)$. Taken together, these findings indicated that overexpression of $H n f 1 b / F o x a 3$ promoted hepatic specification with assistance of extrinsic inducers.

We next investigated whether the HPs could differentiate to HLCs. The HPs were partially digested by trypsin-EDTA, replated on Matrigel-coated dishes, and cultured in HCM that contained OSM, Dex, and HGF for 10 days. Morphologically, the cell population transformed gradually from an extension pattern of short-sided polygons to large polygonal 
flattened cells, which in some cases contained double nuclei (Fig. 3A). In this step we tested the expressions of mature hepatocyte markers by RT-PCR and found that the day 23 HLCs expressed asialoglycoprotein receptor (Asgpr), tyrosine aminotransferase (Tat), glucose-6phosphatase (G6p), tryptophan dioxygenase (Tdo), cytochrome P450 (Cyp) 1A1, and Cyp2B9. The expressions of these genes were not detected in HPs (Fig. 3B). Protein expressions of Alb and Cyp1A1 were confirmed by immunofluorescence staining (Fig. 3C). In an attempt to further characterize the functionality of the induced cells, we evaluated glycogen storage by PAS staining (Fig. 3D). We also observed a biotransforming system in our hepatocyte cells with a test of ICG uptake and release (Fig. 3E). Further, we collected culture medium supernatant at days 13 and 23 and measured Albumin and urea levels by the ELISA and calorimetric assays, respectively. The results showed that HLCs could secrete higher Albumin levels and produce more urea compared to HPs which served as the negative control (Fig. 3F). To show homing and in vivo differentiation, we transplanted HPs intrasplenically into $\mathrm{CCl}_{4}$-treated mice. Prior to transplantation, we labeled the progenitor cells with a fluorescent dye, DiI. Two weeks later, liver samples were examined by immunohistofluorescence staining with antibody against Alb. The presence of double positive cells for DiI and Alb indicated that transplanted cells could integrate and differentiate to hepatic cells in vivo (Fig. 3G). Together, these results supported the result that overexpression of Hnflb/Foxa3 in ES cells under hepatic medium led to the generation of a hepatic fate.

\section{Discussion}

In this study, we have observed that transient expressions of Hnflb, Hnfla, Hnf4a, Foxal, Foxa3, and Hex in mouse ES cells that upregulated $A l b$ mRNA independent of other intrinsic and extrinsic factors. These liver-specific genes are important in endoderm differentiation, 
control of early hepatic growth, coordination of hepatic and cell differentiation necessary to generate a functional liver (for review see $[28,29]$ ). These factors have been used in direct conversion or trans-differentiation of mouse and human fibroblasts to induced HLCs in different studies - Hnf4a plus Foxal, Foxa2 or Foxa3 [5]; Hnfla plus Gata4 and Foxa3, along with inactivation of $19^{\operatorname{Arf}}[6]$; HNF $1 \alpha, H N F 4 \alpha, H N F 6, A T F 5, P R O X 1, C / E B P \beta$ and

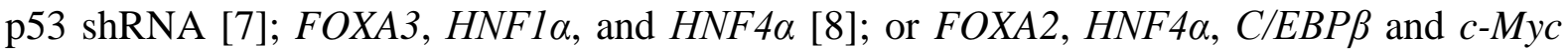
[9] or Hnf4a, Cebpa and Nrli2 [10]. Forced expressions of both Foxa3 and Hnf4a converted rat bone marrow-mesenchymal stem cells to functional HLCs [30].

Interestingly, both Hnflb/Foxa3 out of the six factors in ES cells under hepatic medium enhanced the hepatic fate. Hnflb/Foxa3-ES cells transformed gradually to polygonal flattened cells with prominent nuclei and expressed higher levels of HP markers Alb, Afp, Ttr, $H n f 4 a$, and Dlkl after two weeks compared with the absence of forced expression. Additionally, the HPs further differentiated to HLCs that expressed Asgpr, Tat, G6p, Tdo, Cyp1A1 and Cyp2B9. They showed glycogen storage, ICG uptake and secretion of albumin and urea. The Hnflb/Foxa3-HPs were engraftable and expressed Alb when transplanted into $\mathrm{CCl}_{4}$-treated mice.

Hnflb has an important role in hepatic specification. It has been demonstrated that the expression of Foxa factors is strongly reduced or absent in the presumptive hepatic endoderm of $H n f l b^{-/}$embryos which indicated a requirement of $H n f l b$ for maintaining the expressions of Foxal and Foxa2, specifically in the ventral endoderm, as well as for Foxa3 induction [24]. We observed significant upregulation of Foxa2 after overexpression of Hnflb. The functional Hnflb binding sites in the regulatory sequences of Foxal [24], Foxa2 [31], and Foxa3 [32] have been identified. More importantly, a comprehensive analysis of promoter occupancy during mouse hepatic development demonstrated that Hnflb recruited to the promoter regions of a core group of transcription factors -Foxa2, Hnfla, Hnf4a, Hnf6 and 
Gata6, which comprise autoregulatory and cross-regulatory circuits [31]. Hnflb has a preeminent role in a transcriptional circuit by inducing Hnf4a, Hnf6 [33], Hnfla [31], and Foxa3, [24, 32] and by maintaining Foxa2 expression [31]. On the other hand, the winged helix (FOX) proteins regulate both liver formation and function (for review see [34]). It has been shown that neither Foxal nor Foxa3 is required during early mouse embryogenesis. Both Foxal $^{-/-}$and Foxa3 ${ }^{-/-}$embryos appear normal and develop to birth, however, Foxa2 $2^{-/-}$ has been shown to have a defective hepatic specification $[35,36]$. In contrast, by E10.5, Foxa3 expression is seen in the liver primordium and persists throughout development into the adult liver [27]. Recently, it has been reported that both Hnflb/Foxa3 are sufficient to reprogram mouse fibroblasts into induced hepatic stem cells [37]. Therefore, regulation of liver transcription appears to be controlled by multiple transcription factors acting in concert, [38] where Hnflb/Foxa3 are the "pioneer" factors that have sequence-recognition capability and the ability to bind to a sequence inaccessible to other factors, thus initiating the opening of chromatin at specific sites by recruiting chromatin remodeling factors to their target loci [39].

Taken together, forced expression of both Hnflb/Foxa3 under hepatic conditions enhanced a hepatic lineage fate. Our results have suggested another evidence for the crucial role of these factors in mouse hepatogenesis but research should determine whether these factors could induce human ES cells to a hepatic fate.

\section{Acknowledgments}

This study was funded by grants provided from Royan Institute, the Iranian Council of Stem Cell Research and Technology, the Iran National Science Foundation (INSF), and Iran Science Elites Federation to H.B and Kurdistan University of Medical Sciences to F.F. We express our appreciation to all members of the Hepatocyte Program at Royan Institute for 
their helpful deliberation and consultation during this work. We express our appreciation to Dr. Mohammad Hassan Asghari-Vostikolaee for his assistance in performing animal studies. 


\section{References}

[1] J.A. Thomson, J. Itskovitz-Eldor, S.S. Shapiro, M.A. Waknitz, J.J. Swiergiel, V.S. Marshall, J.M. Jones, Embryonic stem cell lines derived from human blastocysts, Science, 282 (1998) 1145-1147.

[2] C.E. Murry, G. Keller, Differentiation of embryonic stem cells to clinically relevant populations: lessons from embryonic development, Cell, 132 (2008) 661-680.

[3] I.J. Fox, G.Q. Daley, S.A. Goldman, J. Huard, T.J. Kamp, M. Trucco, Stem cell therapy. Use of differentiated pluripotent stem cells as replacement therapy for treating disease, Science, 345 (2014) 1247391.

[4] R.E. Schwartz, H.E. Fleming, S.R. Khetani, S.N. Bhatia, Pluripotent stem cell-derived hepatocyte-like cells, Biotechnol Adv, 32 (2014) 504-513.

[5] S. Sekiya, A. Suzuki, Direct conversion of mouse fibroblasts to hepatocyte-like cells by defined factors, Nature, 475 (2011) 390-393.

[6] P. Huang, Z. He, S. Ji, H. Sun, D. Xiang, C. Liu, Y. Hu, X. Wang, L. Hui, Induction of functional hepatocyte-like cells from mouse fibroblasts by defined factors, Nature, 475 (2011) 386-389.

[7] Y. Du, J. Wang, J. Jia, N. Song, C. Xiang, J. Xu, Z. Hou, X. Su, B. Liu, T. Jiang, D. Zhao, Y. Sun, J. Shu, Q. Guo, M. Yin, D. Sun, S. Lu, Y. Shi, H. Deng, Human hepatocytes with drug metabolic function induced from fibroblasts by lineage reprogramming, Cell stem cell, 14 (2014) 394-403.

[8] P. Huang, L. Zhang, Y. Gao, Z. He, D. Yao, Z. Wu, J. Cen, X. Chen, C. Liu, Y. Hu, D. Lai, Z. Hu, L. Chen, Y. Zhang, X. Cheng, X. Ma, G. Pan, X. Wang, L. Hui, Direct reprogramming of human fibroblasts to functional and expandable hepatocytes, Cell stem cell, 14 (2014) 370-384.

[9] T. Kogiso, H. Nagahara, M. Otsuka, K. Shiratori, S.F. Dowdy, Transdifferentiation of human fibroblasts into hepatocyte-like cells by defined transcriptional factors, Hepatology international, 7 (2013) 937-944.

[10] B. Pournasr, M.H. Asghari-Vostikolaee, H. Baharvand, Transcription factor-mediated reprograming of fibroblasts to hepatocyte-like cells, European journal of cell biology, 94 (2015) 603-610.

[11] S.A. Duncan, Transcriptional regulation of liver development, Dev Dyn, 219 (2000) 131-142.

[12] E. Wandzioch, K.S. Zaret, Dynamic signaling network for the specification of embryonic pancreas and liver progenitors, Science, 324 (2009) 1707-1710.

[13] T. Liu, S. Zhang, D. Xiang, Y. Wang, Induction of hepatocyte-like cells from mouse embryonic stem cells by lentivirus-mediated constitutive expression of Foxa2/Hnf4a, J Cell Biochem, 114 (2013) 2531-2541. 
[14] K. Takayama, M. Inamura, K. Kawabata, K. Katayama, M. Higuchi, K. Tashiro, A. Nonaka, F. Sakurai, T. Hayakawa, M.K. Furue, H. Mizuguchi, Efficient generation of functional hepatocytes from human embryonic stem cells and induced pluripotent stem cells by HNF4alpha transduction, Molecular therapy : the journal of the American Society of Gene Therapy, 20 (2012) 127-137.

[15] M. Inamura, K. Kawabata, K. Takayama, K. Tashiro, F. Sakurai, K. Katayama, M. Toyoda, H. Akutsu, Y. Miyagawa, H. Okita, N. Kiyokawa, A. Umezawa, T. Hayakawa, M.K. Furue, H. Mizuguchi, Efficient generation of hepatoblasts from human ES cells and iPS cells by transient overexpression of homeobox gene HEX, Molecular therapy : the journal of the American Society of Gene Therapy, 19 (2011) 400-407.

[16] D. Nakamori, K. Takayama, Y. Nagamoto, S. Mitani, F. Sakurai, M. Tachibana, H. Mizuguchi, Hepatic maturation of human iPS cell-derived hepatocyte-like cells by ATF5, c/EBPalpha, and PROX1 transduction, Biochemical and biophysical research communications, 469 (2016) 424-429.

[17] X.G. Deng, R.L. Qiu, Y.H. Wu, Z.X. Li, P. Xie, J. Zhang, J.J. Zhou, L.X. Zeng, J. Tang, A. Maharjan, J.M. Deng, Overexpression of miR-122 promotes the hepatic differentiation and maturation of mouse ESCs through a miR-122/FoxA1/HNF4a-positive feedback loop, Liver Int, 34 (2014) 281-295.

[18] A. Kubo, Y.H. Kim, S. Irion, S. Kasuda, M. Takeuchi, K. Ohashi, M. Iwano, Y. Dohi, Y. Saito, R. Snodgrass, G. Keller, The homeobox gene Hex regulates hepatocyte differentiation from embryonic stem cell-derived endoderm, Hepatology, 51 (2010) 633-641.

[19] K. Yamamizu, Y. Piao, A.A. Sharov, V. Zsiros, H. Yu, K. Nakazawa, D. Schlessinger, M.S. Ko, Identification of transcription factors for lineage-specific ESC differentiation, Stem cell reports, 1 (2013) 545-559.

[20] S.N. Hassani, M. Totonchi, A. Farrokhi, A. Taei, M.R. Larijani, H. Gourabi, H. Baharvand, Simultaneous Suppression of TGF-beta and ERK Signaling Contributes to the Highly Efficient and Reproducible Generation of Mouse Embryonic Stem Cells from Previously Considered Refractory and Non-permissive Strains, Stem Cell Rev, 8 (2012) 472481.

[21] Q.L. Ying, J. Wray, J. Nichols, L. Batlle-Morera, B. Doble, J. Woodgett, P. Cohen, A. Smith, The ground state of embryonic stem cell self-renewal, Nature, 453 (2008) 519-523.

[22] C.S. Lee, J.R. Friedman, J.T. Fulmer, K.H. Kaestner, The initiation of liver development is dependent on Foxa transcription factors, Nature, 435 (2005) 944-947.

[23] C.W. Bogue, G.R. Ganea, E. Sturm, R. Ianucci, H.C. Jacobs, Hex expression suggests a role in the development and function of organs derived from foregut endoderm, Dev Dyn, 219 (2000) 84-89.

[24] L. Lokmane, C. Haumaitre, P. Garcia-Villalba, I. Anselme, S. Schneider-Maunoury, S. Cereghini, Crucial role of vHNF1 in vertebrate hepatic specification, Development, 135 (2008) 2777-2786. 
[25] J. Li, G. Ning, S.A. Duncan, Mammalian hepatocyte differentiation requires the transcription factor HNF-4alpha, Genes Dev, 14 (2000) 464-474.

[26] H. Schrem, J. Klempnauer, J. Borlak, Liver-enriched transcription factors in liver function and development. Part I: the hepatocyte nuclear factor network and liver-specific gene expression, Pharmacol Rev, 54 (2002) 129-158.

[27] A.P. Monaghan, K.H. Kaestner, E. Grau, G. Schutz, Postimplantation expression patterns indicate a role for the mouse forkhead/HNF-3 alpha, beta and gamma genes in determination of the definitive endoderm, chordamesoderm and neuroectoderm, Development, 119 (1993) 567-578.

[28] K. Si-Tayeb, F.P. Lemaigre, S.A. Duncan, Organogenesis and development of the liver, Dev Cell, 18 (2010) 175-189.

[29] K.S. Zaret, Regulatory phases of early liver development: paradigms of organogenesis, Nat Rev Genet, 3 (2002) 499-512.

[30] K. Dai, R. Chen, Y. Ding, Z. Niu, J. Fan, C. Xu, Induction of Functional HepatocyteLike Cells by Overexpression of FOXA3 and HNF4alpha in Rat Bone Marrow Mesenchymal Stem Cells, Cells Tissues Organs, 200 (2014) 132-140.

[31] I. Kyrmizi, P. Hatzis, N. Katrakili, F. Tronche, F.J. Gonzalez, I. Talianidis, Plasticity and expanding complexity of the hepatic transcription factor network during liver development, Genes Dev, 20 (2006) 2293-2305.

[32] H. Hiemisch, G. Schutz, K.H. Kaestner, Transcriptional regulation in endoderm development: characterization of an enhancer controlling Hnf3g expression by transgenesis and targeted mutagenesis, EMBO J, 16 (1997) 3995-4006.

[33] A.V. Poll, C.E. Pierreux, L. Lokmane, C. Haumaitre, Y. Achouri, P. Jacquemin, G.G. Rousseau, S. Cereghini, F.P. Lemaigre, A vHNF1/TCF2-HNF6 cascade regulates the transcription factor network that controls generation of pancreatic precursor cells, Diabetes, 55 (2006) 61-69.

[34] J. Le Lay, K.H. Kaestner, The Fox genes in the liver: from organogenesis to functional integration, Physiol Rev, 90 (2010) 1-22.

[35] K.H. Kaestner, H. Hiemisch, G. Schutz, Targeted disruption of the gene encoding hepatocyte nuclear factor 3 gamma results in reduced transcription of hepatocyte-specific genes, Mol Cell Biol, 18 (1998) 4245-4251.

[36] R. Behr, J. Brestelli, J.T. Fulmer, N. Miyawaki, T.R. Kleyman, K.H. Kaestner, Mild nephrogenic diabetes insipidus caused by Foxal deficiency, The Journal of biological chemistry, 279 (2004) 41936-41941.

[37] B. Yu, Z.Y. He, P. You, Q.W. Han, D. Xiang, F. Chen, M.J. Wang, C.C. Liu, X.W. Lin, U. Borjigin, X.Y. Zi, J.X. Li, H.Y. Zhu, W.L. Li, C.S. Han, K.J. Wangensteen, Y. Shi, L.J. Hui, X. Wang, Y.P. Hu, Reprogramming fibroblasts into bipotential hepatic stem cells by defined factors, Cell stem cell, 13 (2013) 328-340. 
[38] D.T. Odom, R.D. Dowell, E.S. Jacobsen, L. Nekludova, P.A. Rolfe, T.W. Danford, D.K. Gifford, E. Fraenkel, G.I. Bell, R.A. Young, Core transcriptional regulatory circuitry in human hepatocytes, Mol Syst Biol, 2 (2006) 20060017.

[39] K.S. Zaret, J. Watts, J. Xu, E. Wandzioch, S.T. Smale, T. Sekiya, Pioneer factors, genetic competence, and inductive signaling: programming liver and pancreas progenitors from the endoderm, Cold Spring Harb Symp Quant Biol, 73 (2008) 119-126. 


\section{Figure legends}

Fig. 1. Hepatic lineage specification in transcription factor-inducible embryonic stem (ES) cells.

(A) Schematic structure of the lentivirus construct. The reverse Tet repressor (rtTA) is encoded by a PGK promoter, binds TRE, and activates transcription in the presence of doxycycline (Dox).

(B) RT-PCR analysis of transduced cells for expressions of Foxa2 at day 7 and (C) Alb at day 13 of induction in basal medium.

(D) Schematic represents the procedure of embryonic stem (ES) cell induction in hepatic/inductive medium. The transduced cells were cultured for 7 days in basal medium in the presence of Dox. Then, enforced cells were treated by bone morphogenetic protein 4 (BMP4) and fibroblast growth factor 4 (FGF4) for three days, followed by hepatocyte growth factor (HGF), insulin-transferrin-selenium (ITS), dexamethasone (Dex), and nicotinamide for another three days to induce hepatic specification.

(E) Relative expression level of Alb mRNA analyzed by RT-PCR in ES cells that overexpressed Hnflb, Hnfla, Hnf4a, Foxal, Foxa3, and Hex gene in hepatic medium.

(F) Phase contrast images of Hnflb and Hnfla overexpressing ES cells at day 13.

(G) Co-immunofluorescent staining of albumin (Alb) with Ck19 for Hnfla and Hnflb ES cells in hepatic medium. The nucleus was stained with DAPI.

Scale bar represents $50 \mu \mathrm{m}$ in G (upper panel) and $100 \mu \mathrm{m}$ in F and G (lower panel)

(H) Forced expressions of Hnflb and Foxa3 together induced hepatic gene expression in basal-cultured condition. Quantitative RT-PCR analyses of Alb and endogenous Hnfla and Hnf4a expressions at day 13.

The data were normalized to Gapdh and original ES cells. The data are presented as mean \pm SD (n=3; ANOVA, Tukey post-hoc test, $* P<0.05$, *** $P<0.001)$. 
Fig. 2. Generation of hepatic progenitors (HPs) by co-forced expression of Hnflb/Foxa3 in hepatic medium at day 13.

(A) Phase contrast morphology of Hnflb/Foxa3 embryonic stem (ES) cells. The cells had an epithelial-like morphology with an extension pattern of short-sided polygons and prominent nuclei. The right panel shows morphology at higher magnification.

(B) The expressions of early hepatic markers in the presence (+) or absence (-) of Dox by qRT-PCR. The data were normalized to Gapdh and original ES cells.

(C) Co-expressions of Alb and Ck19 (upper panel), as well as Hnf4a and Afp (lower panel) in Hnflb/Foxa3 overexpressing ES cells. Nuclei were counterstained with DAPI.

(D) Flow cytometry analysis for Alb expression.

The data are presented as mean $\pm \mathrm{SD}(\mathrm{n}=3 ; * * P<0.01$, **** $P<0.0001$, Student $t$-test $)$. Scale bars: $50 \mu \mathrm{m}$ in A (right panel) and $100 \mu \mathrm{m}$ in other pictures.

\section{Fig. 3. Differentiation of hepatic progenitors (HPs) to hepatocyte-like cells (HLCs).}

Hepatic progenitors (HPs) were further differentiated to mature hepatocyte-like cells (HLCs) in hepatocyte culture medium (HCM) that contained oncostatin M (OSM), dexamethasone (Dex), and hepatocyte growth factor (HGF) for 10 days and evaluated at day 23.

(A) Phase contrast microscopy of differentiated cells that showed large polygonal flattened cells.

(B) Relative expressions of asialoglycoprotein receptor (Asgpr), tyrosine aminotransferase (Tat), glucose-6-phosphatase (G6p), tryptophan dioxygenase (Tdo), cytochrome P450 (Cyp) $1 A 1$, and $C y p 2 B 9$ genes. The data were normalized to Gapdh and original ES cells.

(C) Immunofluorescence staining for Alb; (upper panel) and Cyp1A1 (lower panel). Nucleus was counterstained with DAPI.

(D) Glycogen storage by periodic acid-Schiff (PAS) staining. 
(E) Indocyanine green (ICG) uptake and release.

(F) Albumin secretion levels and urea synthesis were measured by ELISA and calorimetric assays, respectively.

(G) Homing of transplanted cells was detected by Dil and Alb double positive cells in the same section two weeks after transplantation.

HPs were labeled with DiI prior to injection to assess homing of the transplanted cells in carbon tetrachloride $\left(\mathrm{CCL}_{4}\right)$ acute injury liver model. Double DiI/Alb-positive cells were markers of functional activity of the transplanted HPs. The nuclei were counterstained with DAPI.

The data are presented as mean $\pm \mathrm{SD}(\mathrm{n}=3 ; * P<0.05, * * P<0.01, * * * P<0.001, * * * * P<0.0001$, Student $t$-test.). Scale bars: $100 \mu \mathrm{m}$ in D and $50 \mu \mathrm{m}$ in A, C, E and G. 
A 4.00

D

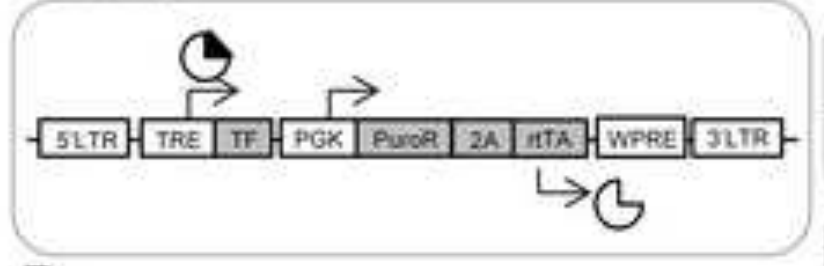

B
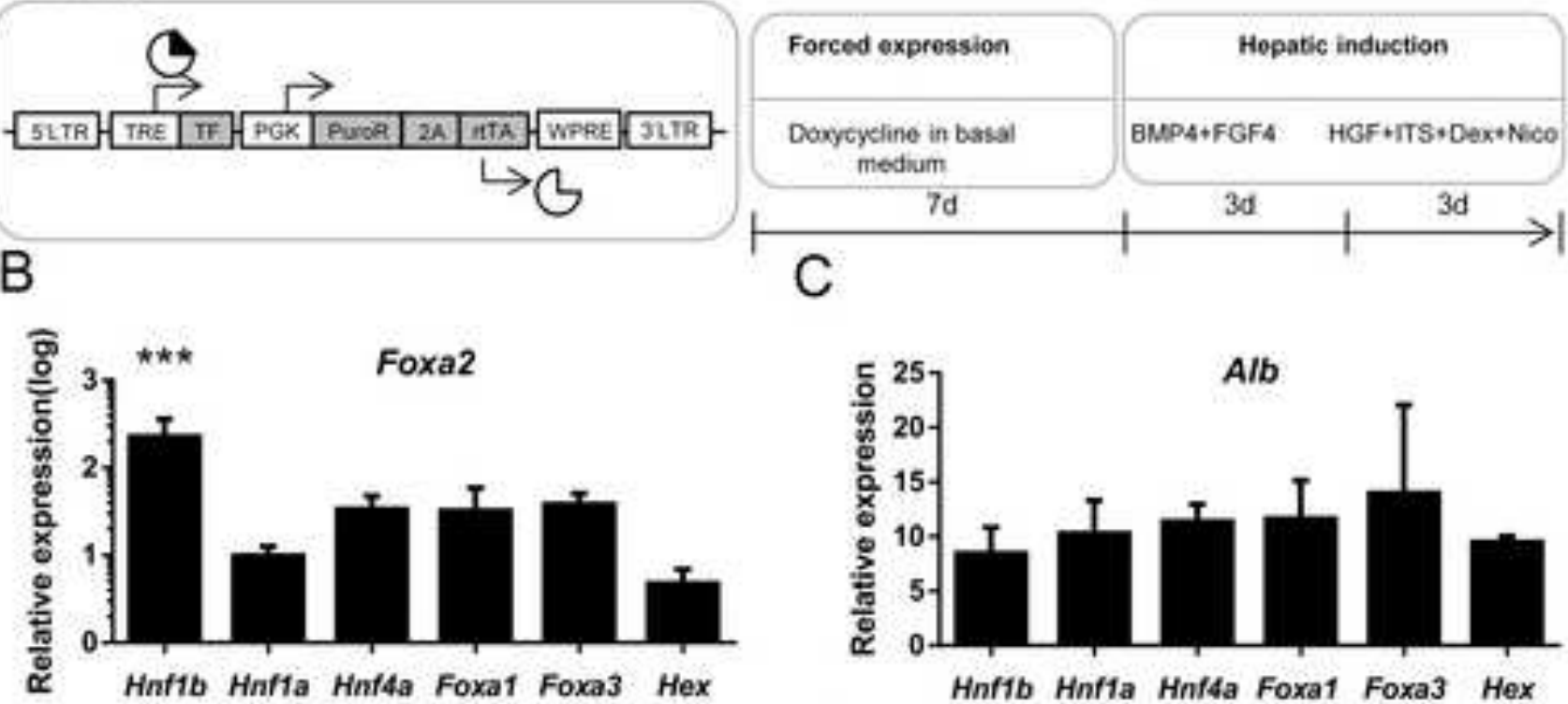

E

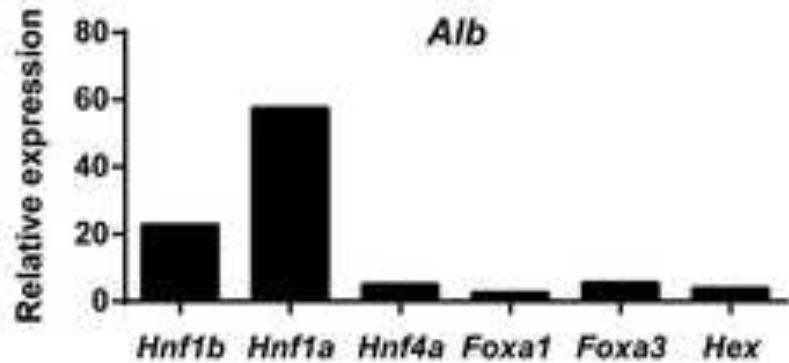

F
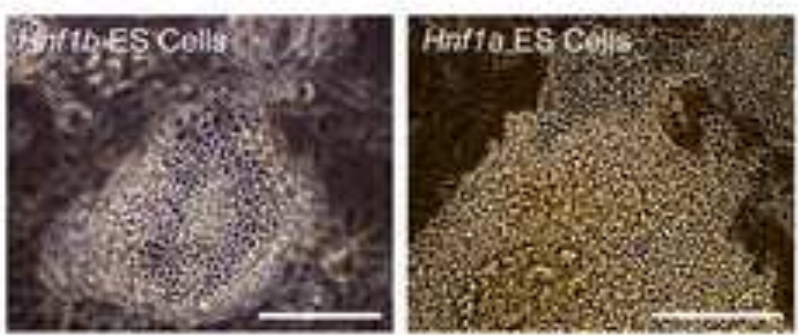

G

Alb
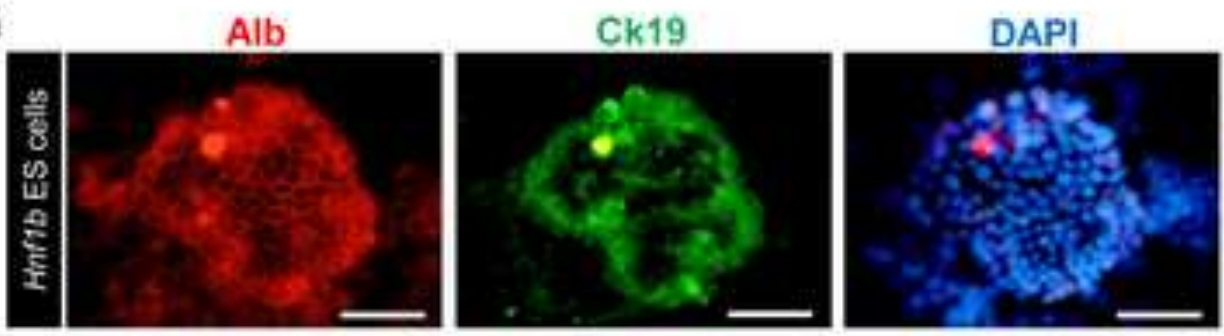

\section{Alb/Ck19/ DAPI}
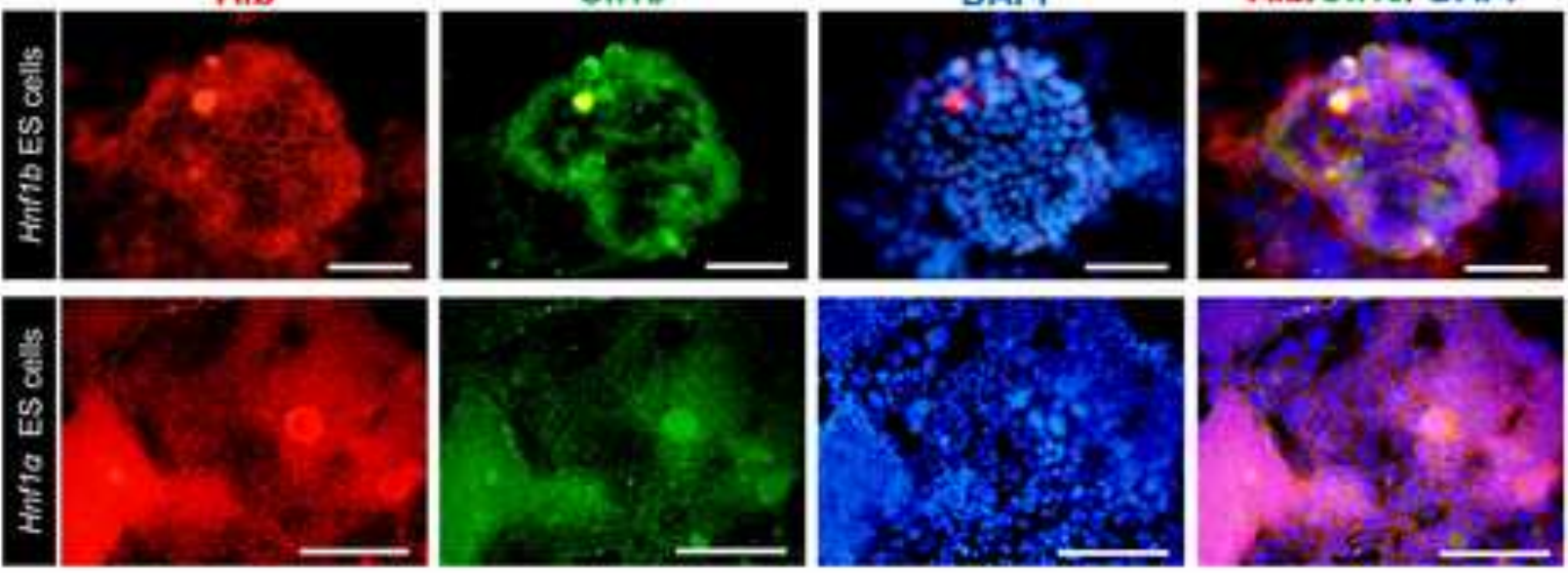

$\mathrm{H}$
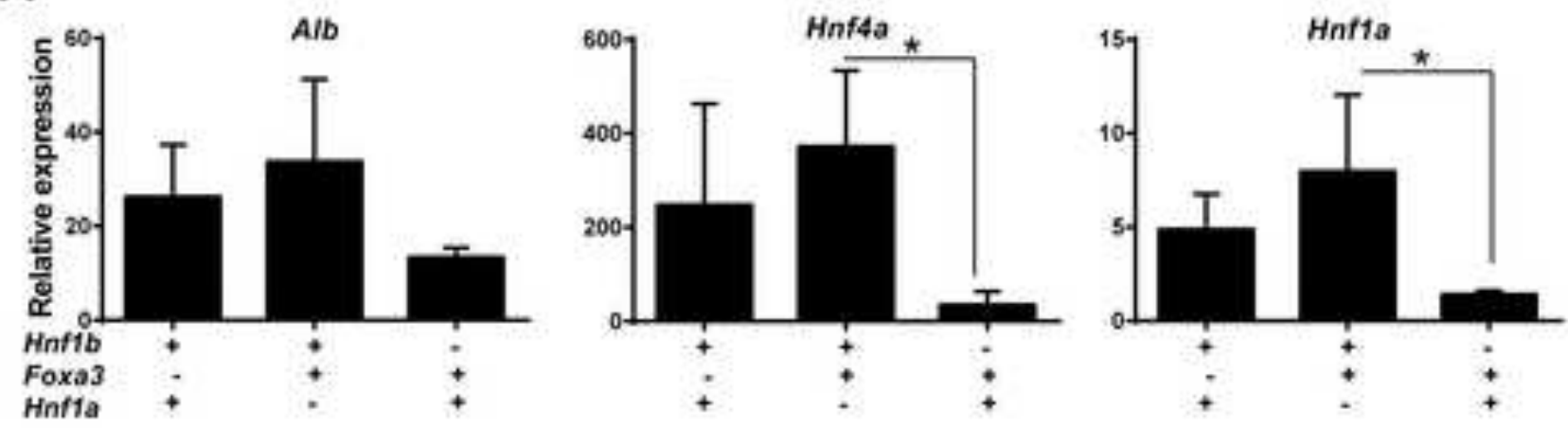


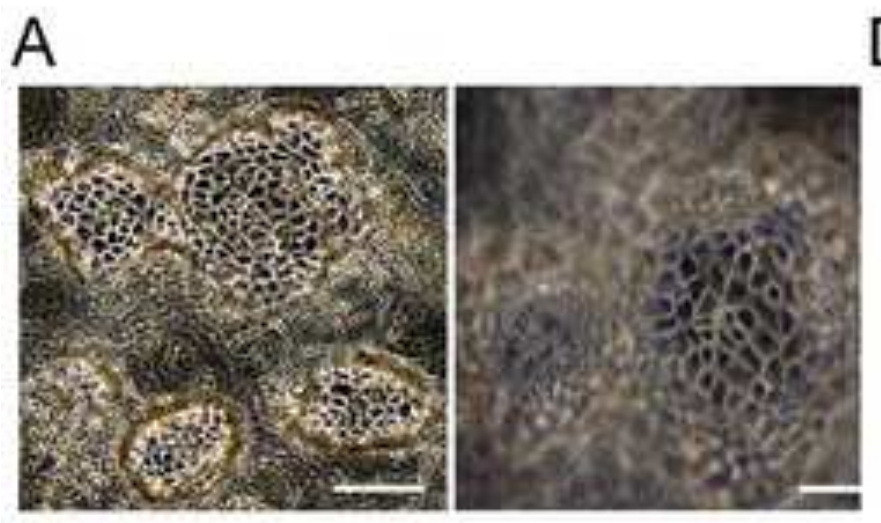

D Induced group

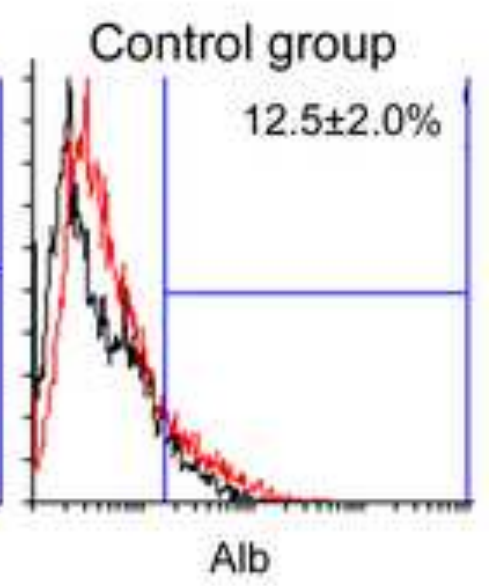

B
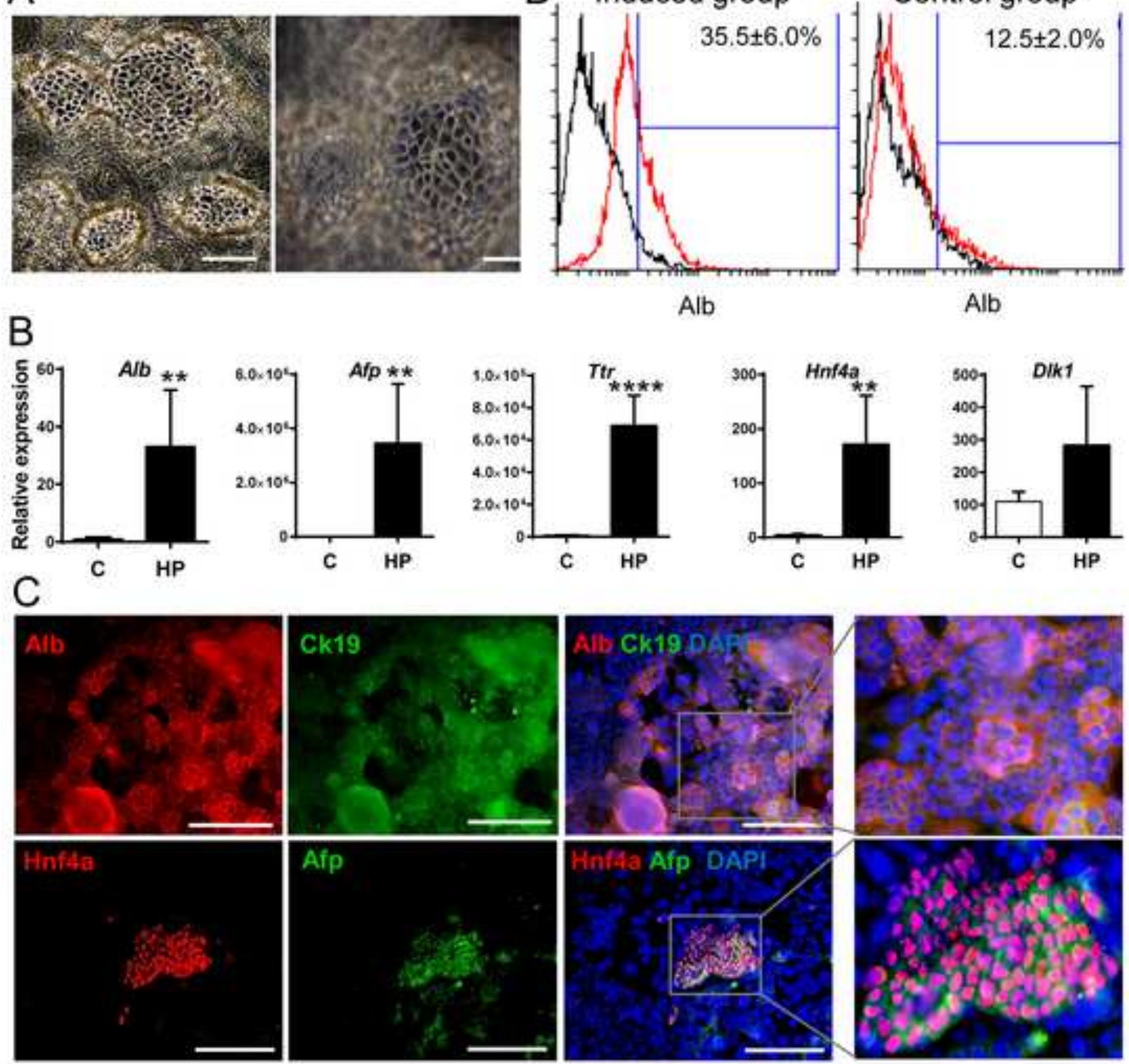


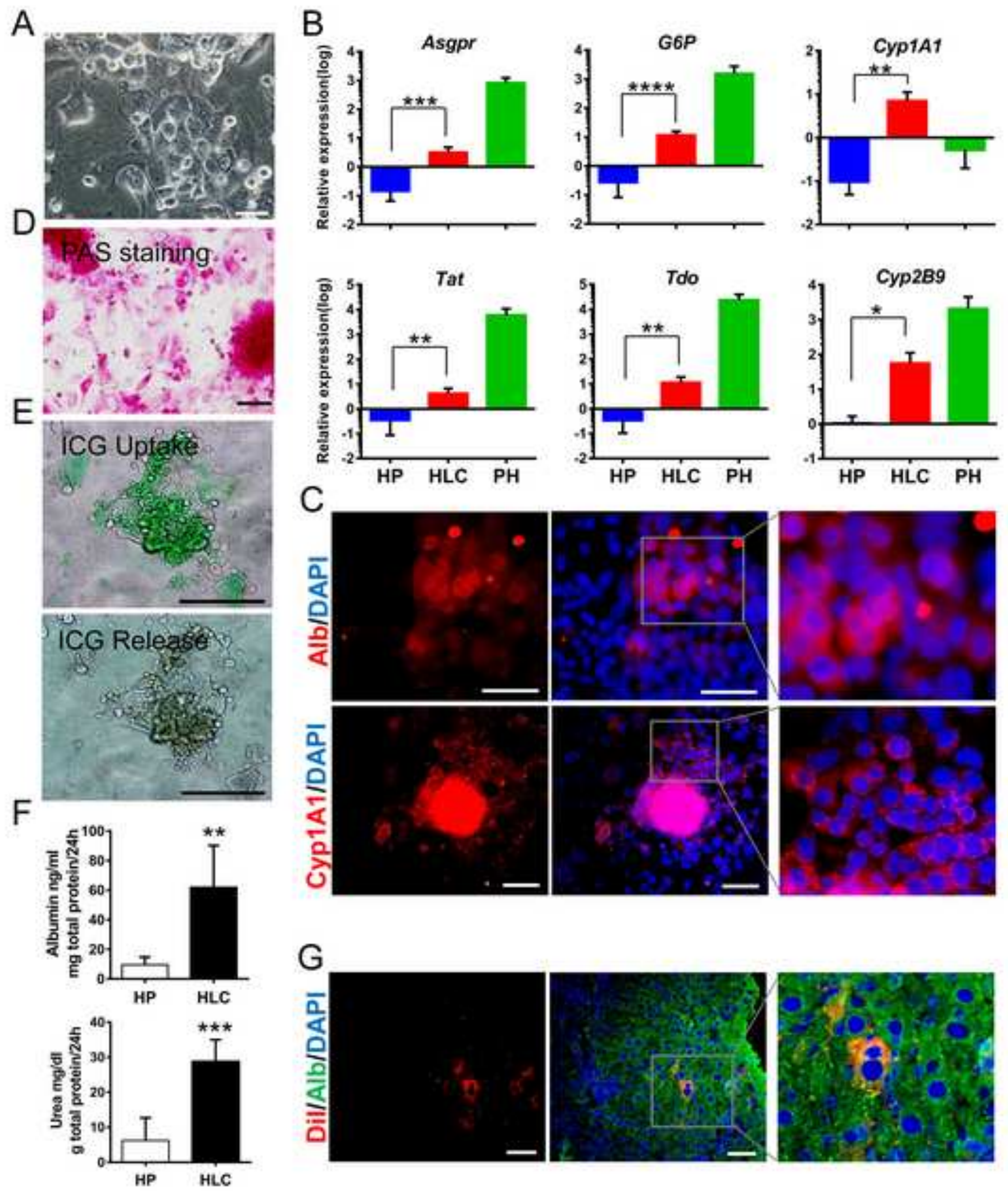


Embryonic stem cells

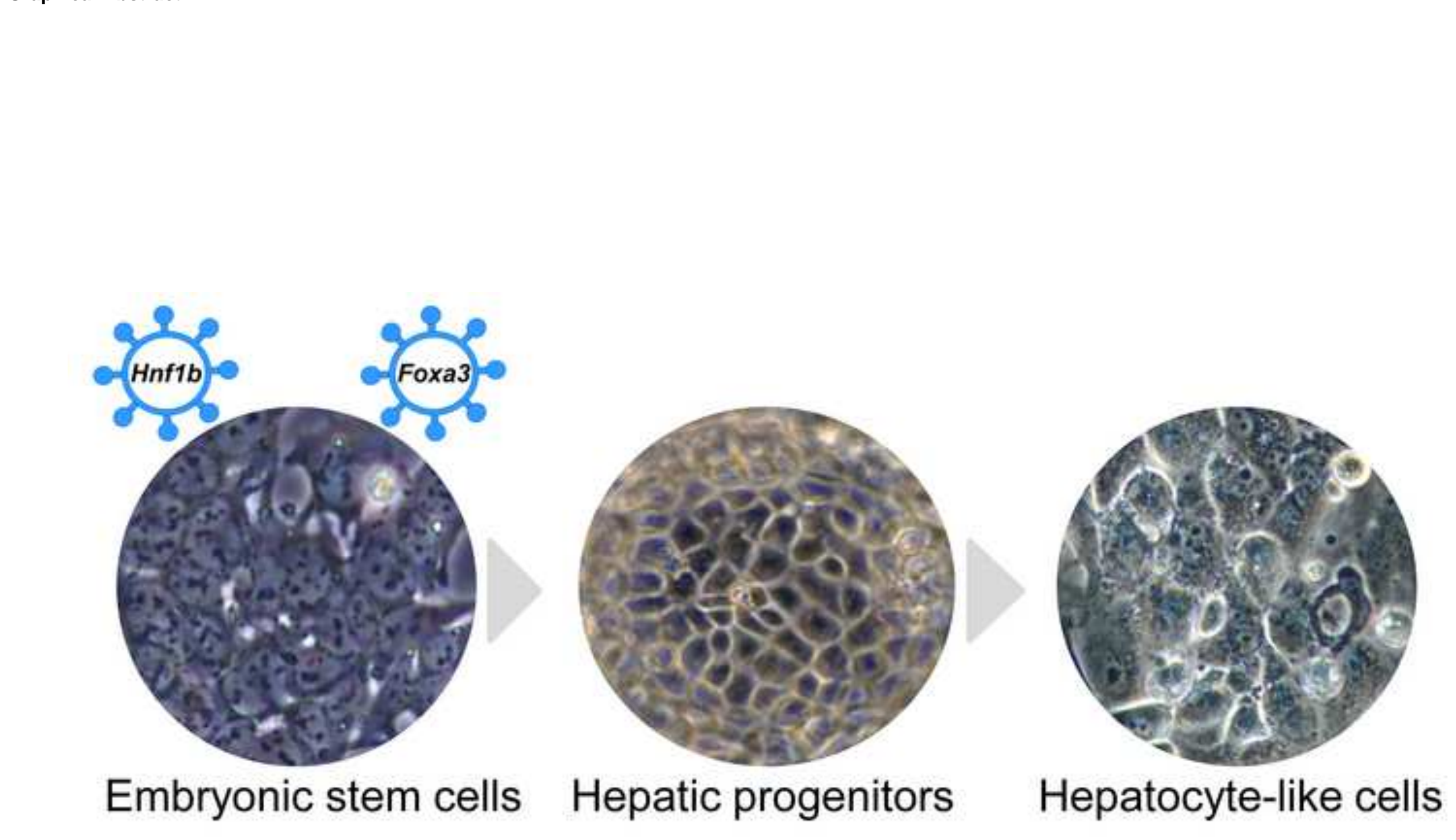

Hepatic progenitors
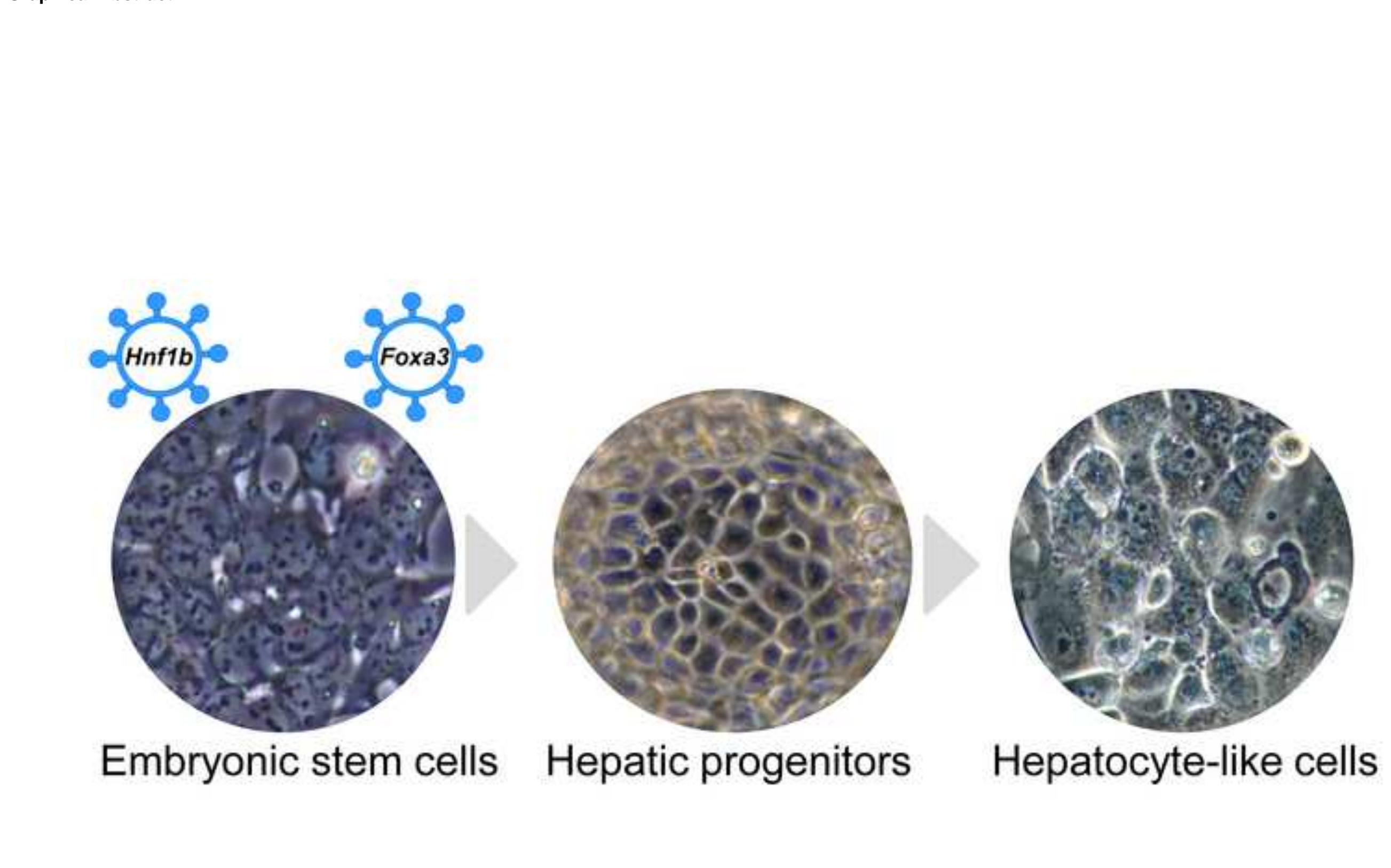

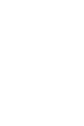
Hepatocyte-like cells Hepatocyte-like cells Hepatic progenitors 